\title{
Quasistatic capillary discharge plasma model
}

\author{
L. C. Steinhauer \\ Redmond Plasma Physics Laboratory, University of Washington, Redmond, Washington 98052, USA
}

\author{
W. D. Kimura
}

STI Optronics, Inc., Bellevue, Washington 98004, USA

(Received 2 February 2006; published 30 August 2006)

\begin{abstract}
A one-dimensional, quasistatic model of a capillary discharge plasma has been developed. Such a plasma is useful as a medium to generate plasma waves for acceleration of electrons via processes such as laser wakefield acceleration or plasma wakefield acceleration. Another important characteristic of the plasma is its intrinsic parabolic density distribution near the center of the capillary, which can channel a laser beam along the capillary. The model is intended to be a design tool to aid in the selection of the capillary parameters in order to obtain desired plasma characteristics, e.g., plasma density and matched laser beam radius for guiding. An optional external axial magnetic field can be included, which improves the laser-channeling effect in some cases. The model also enables a measure of the potential for laser damage of the capillary wall. Results are presented for the design of a gas-filled capillary that will be tested during the staged electron laser acceleration-laser wakefield (STELLA-LW) experiment.
\end{abstract}

\section{INTRODUCTION}

Plasma-based, laser-driven acceleration of electrons requires a suitable plasma medium for creation of the plasma wave (i.e., wakefield) that accelerates the electrons. For example, in plasma wakefield acceleration (PWFA) [1], an ultrashort electron beam ( $e$-beam) pulse passes through the plasma generating plasma waves in its wake. In laser wakefield acceleration (LWFA) [2], an ultrashort laser pulse travels through the plasma and produces the wakefields. These wakefields have demonstrated acceleration gradients $>100 \mathrm{GV} / \mathrm{m}$ [3].

Different methods have been utilized to produce the plasma medium; however, a particularly promising technique is to use a capillary discharge. Figure 1 illustrates the basic geometry of a capillary discharge for PWFA or LWFA applications. It consists of a tube or channel with an inside radius $R_{0}$, typically of order $1 \mathrm{~mm}$ or less. Electrodes are positioned at the ends of the channel so that a high-voltage pulse can be applied across the channel to generate the plasma. This means the channel must be a dielectric material, such as ceramic or plastic, to electrically insulate the two electrodes. Variations of this basic geometry include using a short (e.g., $\sim 3 \mathrm{~mm}$ ) capillary section in tandem with a longer capillary (e.g., $>1 \mathrm{~cm}$ ) whereby the short capillary acts as a trigger for the longer capillary [4]. This reduces the voltage level needed for initial breakdown.

The source of the atoms or molecules for the plasma can be either material ablated from the channel wall or gas that has been injected into the channel prior to the discharge. Examples of the former are polypropylene capillaries operated in vacuum [4]. The electric discharge ablates and ionizes material from the polypropylene wall (primarily hydrogen and carbon atoms). Examples of the latter are hydrogen-gas-filled capillaries featuring ceramic or sapphire channels [5]. Near 100\% ionization of the hydrogen gas occurs when the high voltage is applied.

A quasi-steady-state thermal balance is achieved between the continuous Ohmic heating by the discharge current and thermal conduction loss to the capillary wall. This produces a peaked temperature profile on the axis of the channel. Since the pressure is approximately uniform across the plasma, the density profile has a minimum on axis. This quasiparabolic density profile is suitable for laser channeling.

Once a plasma is formed in the channel, an $e$-beam or laser pulse can be focused into the plasma to generate wakefields, as illustrated in Fig. 1. If the pulse duration

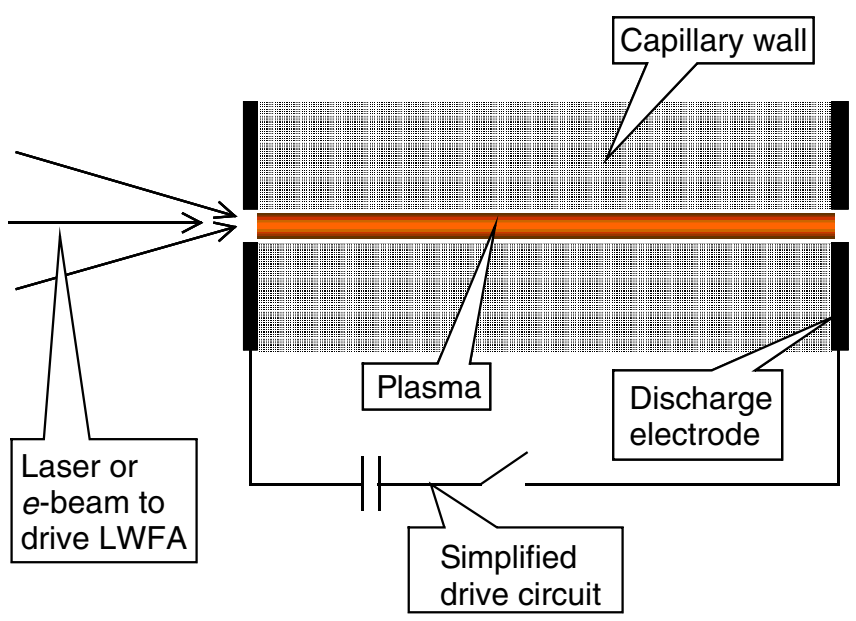

FIG. 1. (Color) Side-view schematic of typical capillary discharge for either LWFA or PWFA applications. 
of the $e$-beam or laser pulse is short enough, then wakefields can be effectively produced via a resonant interaction between the primary Fourier component of the pulse and the plasma frequency. This is referred to as resonant LWFA or resonant PWFA. Since the plasma frequency is dependent on the electron density (or, equivalently, plasma density), this implies the electron density needed in the channel depends on the pulse length of the $e$-beam or laser beam. For example, in LWFA the resonance condition relates the electron density $n_{e}$ and the laser pulse length $t_{L}$ as follows:

$$
n_{e}\left(\mathrm{~cm}^{-3}\right) \approx \frac{3.1 \times 10^{-9}}{t_{L}^{2}(\mathrm{sec})} .
$$

Thus, if $t_{L}=0.5 \mathrm{ps}$, then the required electron density for resonant excitation of the plasma wave is $n_{e} \sim 10^{16} \mathrm{~cm}^{-3}$.

There is another common operating regime called selfmodulated LWFA (SM-LWFA) [6-8], which is a more complex process relying on nonlinear effects occurring between the laser pulse and the plasma. There are also hybrid approaches, such as pseudoresonant LWFA [9], where $t_{L}$ is longer than dictated by Eq. (1), but laser pulse steepening effects occurring within the plasma effectively create Fourier components of a shorter laser pulse so that Eq. (1) is approximately satisfied. Another hybrid is seeded SM-LWFA [10] where a seed $e$-beam pulse creates wakefields via resonant PWFA and the laser pulse immediately follows to amplify the wakefields via SM-LWFA.

All these approaches require specific plasma densities. Hence, often the electron density is predetermined before the capillary parameters are chosen. Furthermore, a minimum laser intensity or charge density is usually needed to drive the wakefield generation process.

For LWFA, maintaining a certain minimum laser intensity impacts the capillary design because of the aforementioned laser-channeling effect produced by the density hollow in the center of the channel $[11,12]$. This makes it possible to maintain the laser intensity over distances much longer than a Rayleigh range. This intrinsic guiding of laser beams is one of the advantages of capillary discharges.

The matching waist size $W_{\mathrm{M}}$ of the laser beam that will be guided down the capillary assuming a parabolic density profile is given by [5]

$$
W_{\mathrm{M}}=\left(\frac{R_{0}^{2}}{\pi r_{e} \Delta n_{e}}\right)^{1 / 4},
$$

where $\Delta n_{e}$ is the depth of the parabolic profile (difference between the density at $r=R_{0}$ and the axis $r=0$ ) and $r_{e}=$ $2.82 \times 10^{-13} \mathrm{~cm}$ is the classical electron radius. Hence, the matching waist size is a function of the ratio of the channel radius and the depth of the density hollow. The depth $\Delta n_{e}$ depends on the thermal conductivity of the plasma. In addition, the amount of energy deposited in the plasma, which affects its formation, is a function of the electrical current passing through the capillary discharge. The voltage across the capillary and the impedance of the discharge are complicated functions of the plasma characteristics.

Thus, many interdependent factors are involved when selecting the parameter values for a capillary design that will achieve target values for $n_{e}$ and $W_{\mathrm{M}}$. Hence, the motivation for the work discussed in this paper was to develop a capillary discharge model that incorporates all the plasma physics and is specifically made to aid in the design of the capillary. This model supports the staged electron laser acceleration-laser wakefield (STELLALW) experiment being performed at the Brookhaven National Laboratory Accelerator Test Facility (BNLATF) [13]. This experiment will be testing the pseudoresonant LWFA [9] and seeded SM-LWFA [10] schemes. For the conditions at the ATF, these methods require relatively low plasma densities $\left(\sim 10^{16}-10^{17} \mathrm{~cm}^{-3}\right)$. Preparations for the STELLA-LW experiment have confirmed that a gas-filled capillary is able to achieve stable densities down to $<10^{16} \mathrm{~cm}^{-3}$. Although STELLA-LW is able to use either polypropylene or gas-filled capillaries, the model presented in this paper applies only to hydrogenfilled capillaries.

Bobrova et al. [14] developed a comprehensive, onedimensional (1D), time-dependent magnetohydrodynamic code for a gas-filled capillary, which agrees well with experimental data [5]. However, a model to assist in the design of a gas-filled capillary need not be time-dependent. The Bobrova computations indicated that a quasi-steadystate is reached fairly quickly (e.g., $\approx 50-80 \mathrm{~ns}$ ). This quasi-steady-state lasts as long as the discharge is maintained (e.g. $\sim 1 \mu \mathrm{s}$ ). Thus, over the short time scale of the LWFA interaction (2-20 ps), the capillary plasma conditions are essentially constant. The quasistatic assumption greatly simplifies the model, reducing it to a coupled set of ordinary differential equations requiring a simple iteration procedure. This permits the model to run efficiently and quickly on a standard-size PC.

The next section describes our 1D, quasistatic capillary discharge model with details of the equations used in the model listed in Appendix A. Section III presents numerical computations for the various design relationships including voltage-current, temperature-current, and density profile. Model predictions for the STELLA-LW experiment are also presented along with a discussion of potential issues with laser damage of the capillary wall. Conclusions are given in Sec. IV.

\section{DESCRIPTION OF 1D, QUASISTATIC CAPILLARY DISCHARGE MODEL}

\section{A. Assumptions and model description}

The development of our model focused on addressing the design needs of the STELLA-LW experiment, i.e., to develop the design relationships that best achieve the de- 
sired properties for laser channeling and the LWFA interaction. These primarily are the target plasma density $n_{0}$ (on axis) and the matched laser spot size $W_{\mathrm{M}}$ for laser guiding. The design task is to link these target parameters to the various externally controllable parameters. The controls are (a) capillary wall radius $R_{0}$, (b) discharge current $I$, (c) hydrogen atomic filling density $n_{f}$, and (d) an optional applied longitudinal (solenoid) magnetic field, $B_{z}$. Note that $n_{f}$ as a control parameter is generally unavailable in polyethylene capillaries where the density is not independently controllable.

The applied external $B$-field is a new control concept we have introduced. Adding a $B$-field (in the direction of the discharge current) reduces the thermal conductivity of the plasma and can steepen both the temperature and density profiles. However, an external $B$-field is currently not being implemented on the STELLA-LW experiment.

Our model relies on the following assumptions:

(1) Large aspect ratio between capillary length and radius. - All plasma and field variables depend only on the radial $r$ coordinate (cylindrical coordinates $r, \theta, z$ are adopted). This $1 \mathrm{D}$ approximation is valid when the capillary length $L$ is much larger than its radius $R_{0}$, so that end effects can be ignored.

(2) Quasi-steady-state.—Quasi-steady-state, i.e., $\partial / \partial t \approx 0$ is assumed. While some radial motion occurs at the initiation of the discharge, this quickly ends as the plasma establishes a balance of radial forces. After the $\sim 50 \mathrm{~ns}$ transient period [14-16], the field and plasma variables become quasistatic, varying slowly in response to changes in the applied electrical current.

(3) Static ions.-Radial force-balance rules out radial ion motion. The static state of the ions means they also have negligible axial and azimuthal motions. This is reasonable since the low ion magnetization for typical capillaries guarantees large ion viscosity, implying that any ion flow will be negligible. Thus, the current density $\boldsymbol{j}$ arises entirely from azimuthal and axial electron flows, i.e., $\boldsymbol{j}=-e n_{e} \boldsymbol{u}$, where $e$ is the electron charge and $\boldsymbol{u}$ is the electron flow velocity.

(4) Quasineutrality. - The ion and electron densities are assumed identical, $n_{e}=n_{i}=n$. This is valid except in a very thin Debye sheath at the capillary wall. Typical Debye lengths are $<1 \mu \mathrm{m}$, whereas capillaries of interest have typical radii of hundreds of microns.

(5) Pure hydrogen as gaseous medium. - The charge of the ions is $Z=1$ and their atomic mass is $A=1$.

(6) Single plasma temperature.-The ions and electrons have identical temperatures, $T_{e}=T_{i}=T$. This approximation is valid since the thermal equilibration time is less than $50 \mathrm{~ns}$ for conditions of interest.
(7) Negligible parasitic losses. — Radiative power loss is neglected, which is appropriate given the tiny plasma volume. Furthermore, charge-exchange losses are neglected since the plasma density is orders of magnitude higher than the expected neutral density.

(8) Braginskii transport is valid.-The classical Braginskii transport rates are adopted [17]. Thermal conduction by both ion and electron species is included, accounting for the magnetizations of each species. Also included are thermoelectric effects, i.e., Nernst effect (a transverse temperature gradient $d T / d r$ giving rise to a friction force against the longitudinal electron current) and Ettinghausen effect (the current flowing across the azimuthal field giving rise to a radial thermal transport).

An added feature in our model, which is not included in the Bobrova model, is the optional externally imposed solenoid magnetic field $B_{z}$. This permits additional control of the discharge properties to complement the azimuthal field $B_{\theta}$ generated by the plasma current. The additional magnetic field reduces the thermal conductivity and steepens the temperature gradient, which tends to deepen the density hollow $\Delta n_{e}$ in Eq. (2). Hence, for fixed $R_{0}$, the external field may allow a smaller matched laser beam waist to be guided. As explained later, this can also reduce the chance of laser beam damage to the channel walls.

Consistent with the foregoing assumptions, the magnetic field and the current density have only axial and azimuthal components: $\boldsymbol{B}=B_{\theta} \hat{\boldsymbol{\theta}}+B_{z} \hat{z}$ and $\boldsymbol{j}=j_{\theta} \hat{\boldsymbol{\theta}}+j_{z} \hat{z}$. In the following, MKS-eV units are used. The steady-state Faraday's law is $\boldsymbol{\nabla} \times \boldsymbol{E}=0$. Its two relevant components are $E_{z}=$ const, and $E_{\theta}=0$. The steady-state Ampere's law $\boldsymbol{\nabla} \times \boldsymbol{B}=\mu_{0} \boldsymbol{j}$ also has two relevant components:

$$
\begin{gathered}
(1 / r) d\left(r B_{\theta}\right) / d r=\mu_{0} j_{z}, \\
d B_{z} / d r=-\mu_{0} j_{\theta},
\end{gathered}
$$

where $\mu_{0}$ is the permittivity of free space. For a static ion fluid, the equation of motion becomes a force-balance relation $\nabla p=(1 / c) \boldsymbol{j} \times \boldsymbol{B}$, with one nontrivial component:

$$
d(n k T) / d r=j_{\theta} B_{z}-j_{z} B_{\theta},
$$

where $p=2 n k T$ is the pressure accounting for both electrons and ions with $Z=1, n$ is the density of electrons, and $k$ is Boltzmann's constant. The right side of Eq. (5) contains the pinch effect, which can be important in some cases.

The electron equation of motion gives rise to Ohm's law $0=-\nabla p_{e}=-e n \boldsymbol{E}+\boldsymbol{j} \times \boldsymbol{B}+\boldsymbol{R}$, which assumes massless electrons and neglects the electron viscosity. Here $\boldsymbol{R}$ is the friction force of the electron density against the stationary background ions. The azimuthal and axial components are of interest: $R_{\theta}=0$; and $R_{z} / e n=E_{z}=$ const. 
For the friction force, the Braginskii transport model is adopted [17]. In the capillary geometry this leads to

$$
\begin{gathered}
\left(\alpha_{\|}^{\prime} b_{\theta}^{2}+\alpha_{\perp}^{\prime} b_{z}^{2}\right) \mu_{0} j_{\theta}+\left(\alpha_{\|}^{\prime}-\alpha_{\perp}^{\prime}\right) b_{\theta} b_{z} \mu_{0} j_{z} \\
-\frac{\beta}{4} \beta_{\Lambda}^{\prime u T} B_{z} \frac{1}{T} \frac{d T}{d r}=0, \\
\left(\alpha_{\|}^{\prime}-\alpha_{\perp}^{\prime}\right) b_{\theta} b_{z} \mu_{0} j_{\theta}+\left(\alpha_{\|}^{\prime} b_{z}^{2}+\alpha_{\perp}^{\prime} b_{\theta}^{2}\right) \mu_{0} j_{z} \\
+\frac{\beta}{4} \beta_{\Lambda}^{\prime u T} B_{\theta} \frac{1}{T} \frac{d T}{d r}=\frac{\mu_{0} E_{z}}{\eta_{\perp 0}},
\end{gathered}
$$

where $\mathbf{b} \equiv \mathbf{B} / B$ is the unit normal in the direction of the magnetic field. The various coefficients $\alpha_{\|}^{\prime}, \alpha_{\perp}^{\prime}, \beta_{\Lambda}^{\prime u T}$, and $\eta_{\perp 0}$ are defined in Appendix A. Here $\eta_{\perp 0}$ is the fully magnetized resistivity, and $\beta \equiv 4 \mu_{0} n k T / B^{2}$ is the local ratio of plasma to magnetic pressures. The energy conservation law is $0=-\boldsymbol{\nabla} \cdot \boldsymbol{q}+\boldsymbol{R} \cdot \boldsymbol{u}$, where $\boldsymbol{q}$ is the thermal heat flux by both ions and electrons. Using Ohm's law we find

$$
(1 / r) d\left(r q_{r}\right) / d r=-E_{z} j_{z}
$$

where the radial heat flux is

$$
q_{r}=\frac{\eta_{\perp 0}}{\mu_{0}} \frac{\beta}{4}\left[\beta_{\Lambda}^{\prime u T}\left(B_{z} j_{\theta}-B_{\theta} j_{z}\right)-\left(\kappa_{e}^{\prime}+\kappa_{i}^{\prime}\right) \frac{B^{2}}{\mu_{0}} \frac{1}{T} \frac{d T}{d r}\right] .
$$

The coefficients $\kappa_{i}^{\prime}$ and $\kappa_{e}^{\prime}$ are given in Appendix A. The foregoing constitute a complete system of ordinary differential equations for the variables as a function of $r$.

\section{B. Model parameters}

From the capillary designer's viewpoint, the controllable design parameters are $R_{0}, L, n_{f}, I$ (to the extent allowed by the high-voltage circuit), and $B_{z}$. The length of the capillary is set by other considerations, such as the dephasing length of the plasma waves, and is not considered in this model except for the assumption that it be much longer than $R_{0}$.

The hydrogen gas-fill pressure is proportional to the prefill density of atomic hydrogen in the capillary, which is identical to the subsequent average density

$$
\langle n\rangle=\left(2 / R_{0}^{2}\right) \int_{0}^{R_{0}} n r d r .
$$

In principle, the discharge current passing through the capillary is set by both external circuit parameters and the impedance of the discharge. In practice, controlling the discharge current by varying the external circuit parameters is not always straightforward. The current is given by

$$
I=\left(2 \pi / \mu_{0}\right) R_{0} B_{\theta}\left(R_{0}\right) .
$$

For the optional external $B$-field we shall assume a value of $1 \mathrm{~T}$, which is representative of the upper limit obtainable from room-temperature magnets in this type of application. As an upper limit, it serves to illustrate the maximum effect the external field would have on the capillary characteristics.

The radius of the capillary compared to the radius of the matched laser beam can be expressed as a ratio $R_{0} / W_{\mathrm{M}}$. This ratio can serve as an index related to the potential for laser damage to the capillary wall. Even if $W_{m}<R_{0}$, the outer edges of the laser beam can still strike the capillary wall. A simple rule of thumb is to have $R_{0} / W_{\mathrm{M}}$ exceed 2 or 3 so that the laser field at the wall is much less than at the axis. If $R_{0} / W_{\mathrm{M}}<2$, then the possibility of laser damage of the wall is considerably increased.

\section{Model algorithm method}

The mathematical description of the capillary consists of five coupled ordinary differential equations in $r$ on the domain $\left[0, R_{0}\right]$ for the variables $B_{\theta}, B_{z}, n, T$, and $q_{r}$, i.e., Eqs. (3)-(5), (8), and (9). The other equations serve as auxiliary ones. The system contains one explicit input parameter, $E_{z}$. All other inputs are associated with the boundary conditions of the system. Some of the boundary conditions are applied at the axis $(r=0)$ and some at the capillary wall $\left(r=R_{0}\right)$. The axis conditions are $B_{\theta}(0)=0$, $n(0)=n_{0}$, and $q_{r}(0)=0$, where $n_{0}$ is the target on-axis density. The wall conditions are $B_{z}\left(R_{0}\right)=B_{z w}$ (externally applied field) and $T\left(R_{0}\right)=T_{w}$ (wall temperature). The condition $T_{w}=0$ has generally been assumed [14-16]. In our modeling, $T_{w}=1 \mathrm{eV}$ is assumed in order to account roughly for the presence of an electrostatic sheath at the wall. However, the results are insensitive to this assumption.

The foregoing system can be solved by a shooting method. Treating it as an initial value problem, the values $B_{z}(0)$ and $T(0)$ are guessed and the equations integrated by a 4th-order Runge-Kutta algorithm. Then the guessed values are adjusted using the Newton-Raphson method, and the integration repeated until the desired wall conditions $B_{z}\left(R_{0}\right)=B_{z w}$, and $T\left(R_{0}\right)=T_{w}$ are achieved. The computational procedure is coded in Visual Basic imbedded in EXCEL software.

\section{COMPARISON WITH BOBROVA MODEL AND SAMPLE MODEL RESULTS}

\section{A. Comparison of quasistatic model with Bobrova simulation}

The accuracy of our quasistatic model was verified by comparing its predictions with the fully time-dependent simulation of Bobrova et al. [14]. As listed in Table I, the on-axis plasma temperature and plasma density $n_{0}$ were chosen to match the Bobrova calculation. The predictions of the two models are compared in Fig. 2, where the Bobrova result (solid black curve) is taken from Fig. 3 of Ref. [14] and represents the computed density profile at 
TABLE I. Input parameters used in quasistatic model for comparison with Bobrova model [14].

\begin{tabular}{ll}
\hline \hline \multicolumn{1}{c}{ Input parameter } & \multicolumn{1}{c}{ Value } \\
\hline Capillary inner radius, $R_{0}$ & $150 \mu \mathrm{m}$ \\
On-axis plasma temperature, $T(0)$ & $6.19 \mathrm{eV}$ \\
Plasma temperature at capillary wall, $T\left(R_{0}\right)$ & $1 \mathrm{eV}$ \\
On-axis plasma density, $n_{0}(0)$ & $2.26 \times 10^{18} \mathrm{~cm}^{-3}$ \\
\hline \hline
\end{tabular}

$t=80 \mathrm{~ns}$ corresponding to an approximate steady-state condition. Also shown is the density profile predicted by the quasistatic model (dashed red curve). There is excellent agreement between the two models except very close to the capillary wall. The differences there arise because the Bobrova model took into account neutral hydrogen that appears in the cold layers near the wall. This agreement between the quasistatic model and the fully timedependent computation was achieved without any parametric adjustments.

The shape of the plasma density profile in the central portion of the capillary is critical since this determines the guiding of the laser beam. As seen in Fig. 2, both models agree in this region. Note that both models predict a superparabolic density rise outside the core $(r>75 \mu \mathrm{m})$.

The discharge currents predicted by the models can also be compared. Bobrova et al. used a current pulse given by $I(t)=I_{0} \sin \left(\pi t / t_{0}\right)$, where $I_{0}=250 \mathrm{~A}$ and $t_{0}=200 \mathrm{~ns}$. At $t=80 \mathrm{~ns}$, this equation yields $I=211 \mathrm{~A}$, which is in close agreement with the quasistatic model value of $I=$ 216 A.

In the quasistatic model results that follow, one further comparison is made. In addition to the fully time-

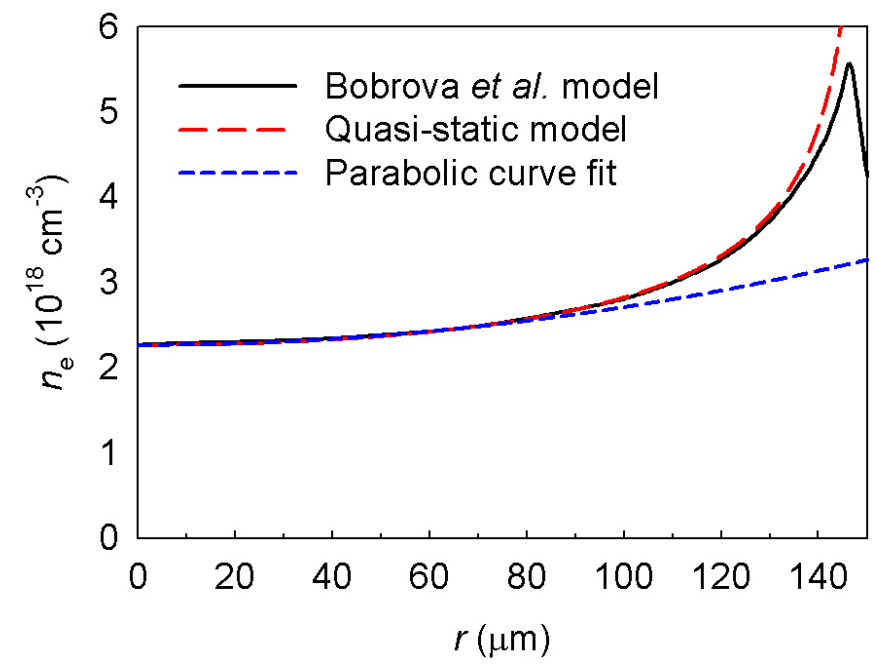

FIG. 2. (Color) Comparison of model predictions for plasma density versus radius. Plotted is the result given in Fig. 3 of Ref. [14] at $80 \mathrm{~ns}$ (solid curve) and the quasistatic model prediction (red long-dashed curve). A parabolic fit to the inner portion $(r<75 \mu \mathrm{m})$ is also shown (blue short-dashed curve). dependent analysis, Bobrova et al. [14] devised a simple analytic model of the capillary. Their analytic model has the advantage of giving simple algebraic relationships between key parameters; however, in doing so it makes certain assumptions and excludes parts of the physics. Details of the analytic model, including a minor modification made for this paper, are summarized in Appendix B. Comparing the analytic model results with the quasistatic model helps reveal where the simplifications of the analytic model impact its accuracy.

\section{B. Capillary discharge properties predicted by quasistatic model}

For the conditions at the ATF, the seeded SM-LWFA experiment and pseudoresonant LWFA experiments require nominal plasma densities of $8.9 \times 10^{16} \mathrm{~cm}^{-3}$ and $1.1 \times 10^{16} \mathrm{~cm}^{-3}$, respectively. For the purposes of exploring the capillary discharge behavior using our quasistatic model, we shall assume an intermediate reference density of $n_{0}=4 \times 10^{16} \mathrm{~cm}^{-3}$, and a reference capillary radius of $R_{0}=250 \mu \mathrm{m}$.

As an element in an electrical circuit, the capillary acts as a nonlinear resistor. The electrical resistance of the capillary discharge is

$$
\mathcal{R}=E_{z} L / I
$$

Figure 3 shows the electric field $E_{z}(=\mathcal{R} I / L)$ versus the current $I$. The nonlinearity of the capillary resistivity is quite evident as it deviates from the straight dot-dashed lines shown on the plot that represent linear resistance. Also shown is the analytic model result, which predicts $E_{z} \propto I^{2 / 5}$ [see Eq. (B2)]. This unusual scaling arises because the resistance decreases with temperature, $\eta \propto$ $T^{-3 / 2}$, and $T$ increases with current. The presence of a

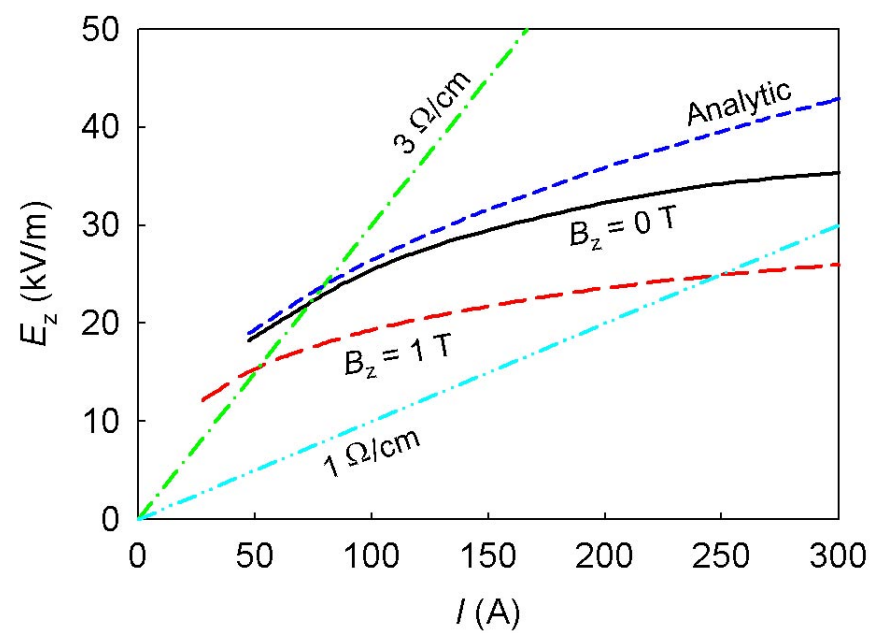

FIG. 3. (Color) Quasistatic model predictions for electric field gradient versus the capillary discharge current with $R_{0}=$ $250 \mu \mathrm{m}$ and $n_{0}=4 \times 10^{16} \mathrm{~cm}^{-3} . B_{z}$ is the external solenoid field. 
1-T solenoid field reduces the resistance by $20 \%-25 \%$ because it decreases the thermal conductivity of the plasma, enabling the temperature to run higher. Note that while the analytic model gives a reasonable portrayal of the $E_{z}$ vs $I$ scaling (i.e., the $\sim I^{2 / 5}$ dependence), its constant of proportionality is $25 \%-100 \%$ too high depending on the value of $I$ and $B_{z}$.

The on-axis plasma temperature $T_{0}$ depends on the discharge current $I$. Figure 4 shows the effect of discharge current and applied solenoid field on $T_{0}$. The scaling $T_{0} \sim$ $I^{2 / 5}$ predicted by the analytic model [see Eq. (B1)] is for the case with no solenoid field. In predicting $T_{0}$, the analytic model yields a constant of proportionality closer to the quasistatic model. When a solenoid field is added, the temperature rises sharply.

Without the solenoid field, we note that $T_{0}$ is in the same range, 3-7 eV, observed in discharges at much higher plasma densities modeled elsewhere [14-16]. These temperatures are high enough that the fully ionized approximation is valid. This near-complete ionization in gas-filled capillaries has been experimentally verified elsewhere [18].

The central hollow in the plasma density in the center of the capillary is crucial for guiding the laser beam. However, complicating this is the pinch effect. If pinching is negligible, then the pressure profile is uniform and the falling temperature toward the capillary wall guarantees a rising density. Negligible pinch effect is a very good approximation for capillary discharges at high plasma densities, e.g., $\geq 10^{18} \mathrm{~cm}^{-3}$ [14-16]. However, at low densities, e.g., $10^{16}-10^{17} \mathrm{~cm}^{-3}$, it can be more important depending on the amount of discharge current. The pinch effect pushes the plasma inward from the wall, thereby causing the pressure to fall near the wall. This pulls the density down near the edge, diminishing the laser guiding effect.

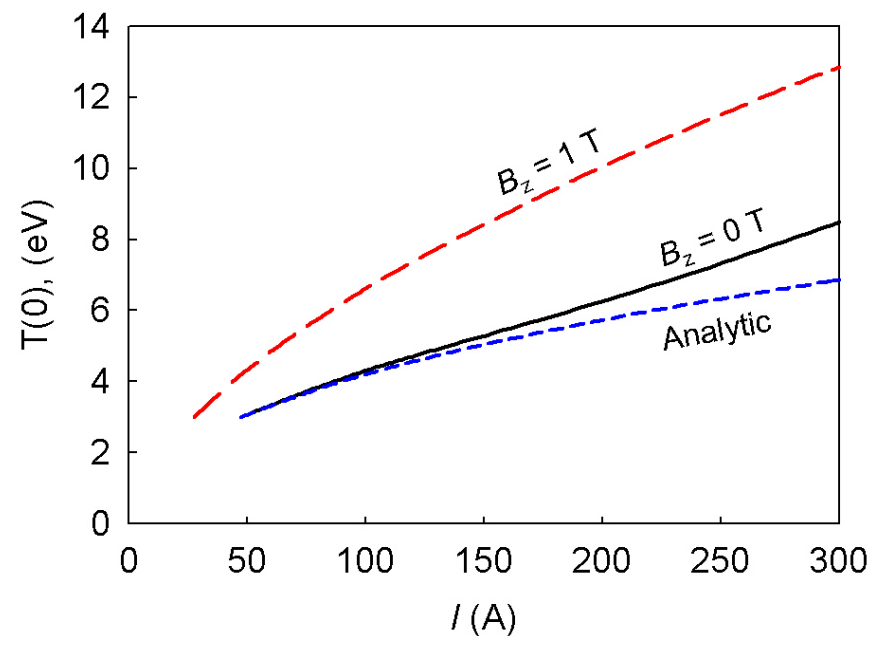

FIG. 4. (Color) Quasistatic model predictions for on-axis temperature versus capillary discharge current with $R_{0}=250 \mu \mathrm{m}$ and $n_{0}=4 \times 10^{16} \mathrm{~cm}^{-3} . B_{z}$ is the external solenoid field.
Figure 5 shows the computed density and pressure profiles for a fixed current $I=100 \mathrm{~A}$. The pinch effect is beginning to manifest itself as evident by the fall in pressure near the wall. Recall the analytic model does not include any pinch effect, hence, its pressure is constant with radius and the density profile follows the nominal quasiparabolic shape. With the pinch effect, the quasistatic model indicates a slight altering of the parabolic profile with and without the solenoid $B$-field, but in this case it is not enough to be significant. At higher currents, e.g., $I=$ $300 \mathrm{~A}$, the pinch effect can be strong enough that the density in the center of the plasma actually has an inverted profile with a slight maximum on axis.

Figure 6 shows the damage index ratio $R_{0} / W_{\mathrm{M}}$ predicted by the quasistatic and analytic models as a function of plasma current and solenoid field. For the analytic approximation, the spot size given by Eq. (B3) leads to an expression for the simple damage index of $R_{0} / W_{\mathrm{M}}=$ $1.35\left(R_{0} / \lambda_{p 0}\right)^{1 / 2}$, where $\lambda_{p 0}$ is the plasma wavelength at the axis. Note that the analytic model with its limited physics is the most pessimistic. The quasistatic model predicts higher ratios with the solenoid $B$-field giving the highest index values for the range of currents shown.
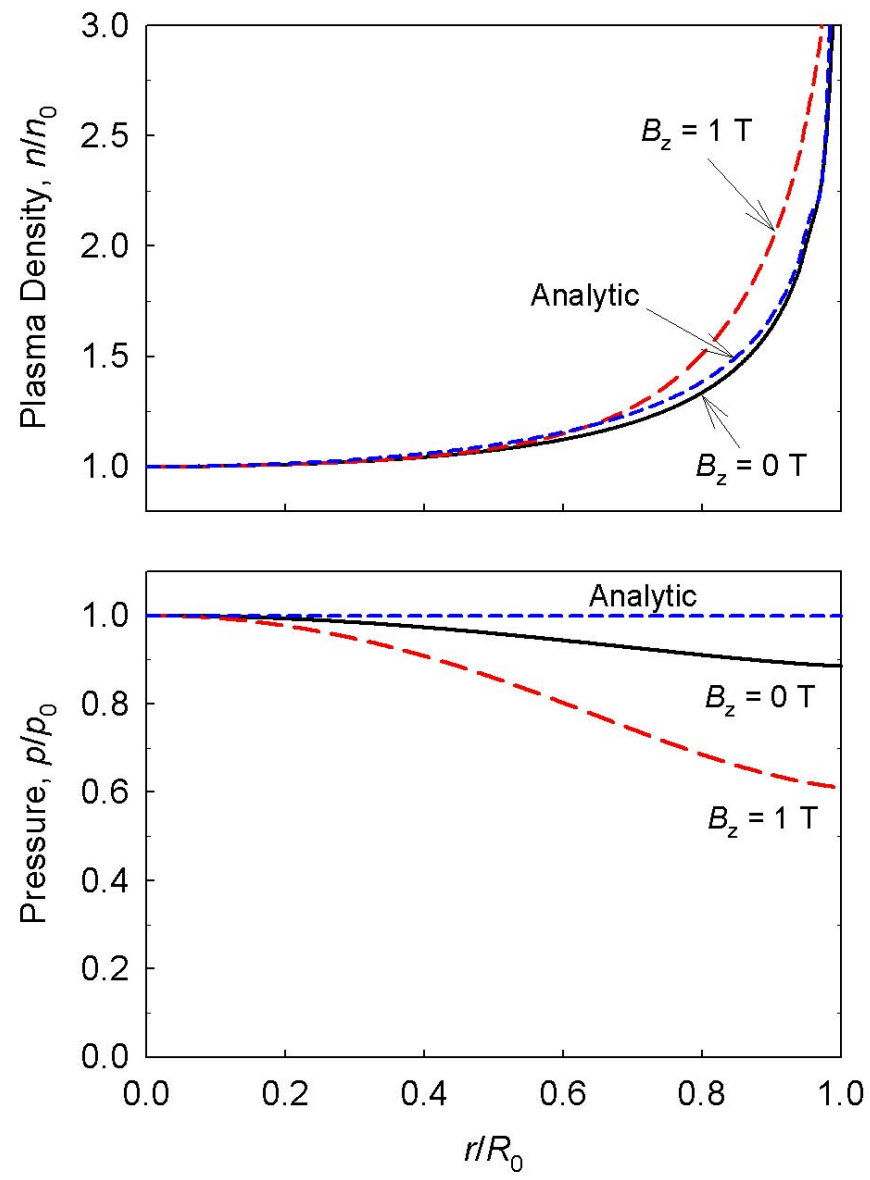

FIG. 5. (Color) Quasistatic model predictions for plasma density and pressure versus radius for $I=100 \mathrm{~A}$ with $R_{0}=250 \mu \mathrm{m}$ and $n_{0}=4 \times 10^{16} \mathrm{~cm}^{-3} . B_{z}$ is the external solenoid field. 


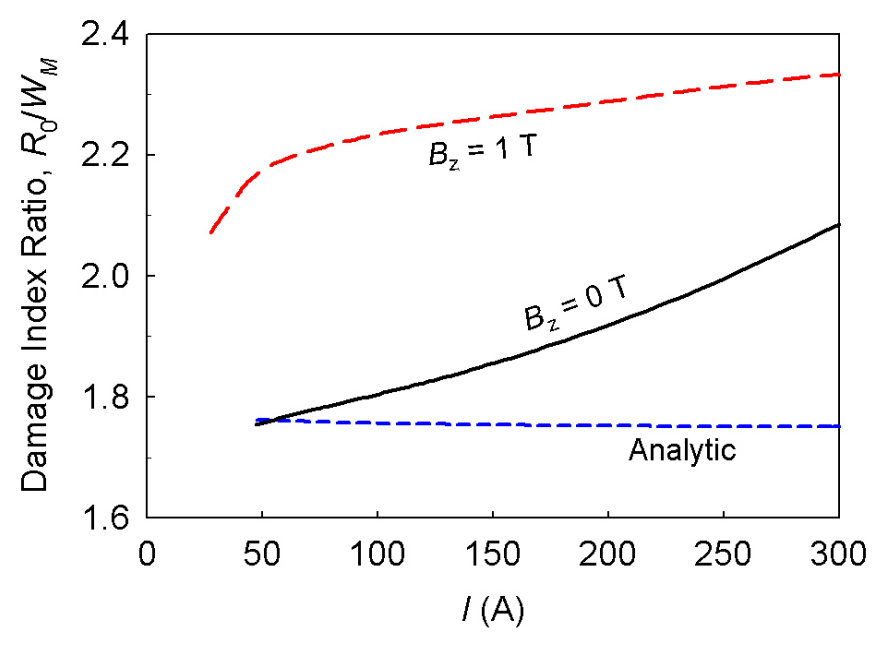

FIG. 6. (Color) Quasistatic model predictions for damage index ratio versus current with $R_{0}=250 \mu \mathrm{m}$ and $n_{0}=$ $4 \times 10^{16} \mathrm{~cm}^{-3} \cdot B_{z}$ is the external solenoid field.

The density profile near the capillary wall is "superquadratic," in that $n$ rises much faster than $r^{2}$ near the wall as shown in Fig. 2. This edge layer serves to shield the capillary wall from the laser beam in the sense that it will reduce the field in this region because of its higher refractive index.

Thus, the simple $R_{0} / W_{\mathrm{M}}$ damage criterion can be enhanced to reflect the nonparabolic nature of the density profile near the wall. An improved damage index can be devised based on the actual maximum electric field at the wall surface $\left(r=R_{0}{ }^{+}\right)$. Consider the following improved definition for the damage index:

$$
D \equiv \ln \left[E_{0} /\left|E_{r}^{2}+E_{z}^{2}\right|_{r=R_{0}^{+}}\right],
$$

which represents the number of $e$-folds that the field falls between the axis and the wall surface. As an example, for a pure Gaussian beam where the plasma field $E\left(R_{0}{ }^{-}\right)$and the wall field $E\left(R_{0}{ }^{+}\right)$are assumed to be identical, the damage index is $D=D_{\text {Gaussian }}=\left(R_{0} / W_{\mathrm{M}}\right)^{2}$, i.e., the square of the simple damage index. Because the density profile in the channel is not purely parabolic, the laser profile in the channel will not be purely Gaussian. A method for computing the actual, non-Gaussian laser profile and the enhanced damage index $D$ is described in Appendix C.

\section{Predictions for STELLA-LW experiment}

To illustrate the application of the quasistatic model to LWFA experiments, Table II lists the model results for conditions representative of the planned STELLA-LW pseudoresonant LWFA and seeded SM-LWFA experiments with the exception of the solenoid $B$-field. The results in Table II assume a $0.3 \mathrm{~T}$ solenoid field; whereas, the STELLA-LW LWFA experiments are not presently using an external $B$-field.

The average plasma densities $\langle n\rangle$ in Table II are what are needed to achieve the target on-axis densities for the pseudoresonant LWFA and seeded SM-LWFA experiments (see Sec. III B).

For the $2.5 \mathrm{TW}$ peak power that is anticipated to be available from the ATF $\mathrm{CO}_{2}$ laser, the pseudoresonant LWFA experiment requires a matching laser waist size of $W_{\mathrm{M}}=145 \mu \mathrm{m}$. To achieve this, the quasistatic model indicates the channel radius must be $R_{0}=160 \mu \mathrm{m}$ with $I=17 \mathrm{~A}$. This is the result of the much weaker refractive power of low-density plasmas. This yields a very low ratio for $R_{0} / W_{\mathrm{M}}$.

For the seeded SM-LWFA experiment, the laser peak power will be $\sim 0.5 \mathrm{TW}$, which is sufficient for amplifying the wakefield produced by the seed $e$-beam pulse. The laser beam spot size $(157 \mathrm{~mm})$ is still comparable to the pseudoresonant case. However, although the laser beam size is similar, the higher plasma density of the seeded SM-LWFA experiment means the required channel size is much larger, i.e., $R_{0}=450 \mu \mathrm{m}$ and the current can be higher, i.e., $I=$ $158 \mathrm{~A}$. The larger channel radius means $R_{0} / W_{\mathrm{M}}$ is close to 3 for the seeded SM-LWFA experiment, which implies that laser damage of the capillary will probably not be an issue.

Table II also shows the damage index $D$ based on the actual computed fields. The range of displayed values for $D$ corresponds to the range of wall skin depths $\delta_{w}=2 \lambda$ to $0.5 \lambda$, where $\lambda$ is the laser wavelength. A range is needed since the dielectric constant at $\lambda=10.6 \mu \mathrm{m}$ of the capillary wall material (i.e., Macor ${ }^{\mathrm{TM}}$ machinable ceramic) used during the STELLA-LW experiment is unknown. For comparison, Table II also gives the damage index if the laser profile retained a Gaussian profile to the edge, i.e., $D_{\text {Gaussian }}=\left(R_{0} / W\right)^{2}$. For the pseudoresonant LWFA case, $D$ has values approaching 3 , which is a considerable improvement over the simple damage index ratio of 1.25 assuming a Gaussian beam.

\section{CONCLUSION}

The quasistatic capillary model has proven to be a very useful and easy-to-use design tool. Its comprehensive inclusion of the plasma physics and fluid equations, and its agreement with the fully time-dependent model of

TABLE II. Capillary results and predictions for STELLA-LW experiment assuming an external B-field of $0.3 \mathrm{~T}$.

\begin{tabular}{lcccccccr}
\hline \hline LWFA Method & $B_{z w}(\mathrm{~T})$ & $\langle n\rangle\left(10^{17} \mathrm{~cm}^{-3}\right)$ & $R_{0}(\mu \mathrm{m})$ & $T_{0}(\mathrm{eV})$ & $I(\mathrm{~A})$ & $R_{0} / W_{\mathrm{M}}$ & $D_{\text {Gaussian }}$ & $D$ \\
\hline Pseudoresonant LWFA & 0.3 & 0.165 & 160 & 3.3 & 17 & 1.1 & 1.25 & $2.0-3.3$ \\
Seeded SM-LWFA & 0.3 & 1.29 & 450 & 4 & 158 & 2.87 & 9.9 & $7.7-8.7$ \\
\hline \hline
\end{tabular}


Bobrova, gives us confidence in its accuracy. The model has been used to design the STELLA-LW capillaries for two different LWFA experiments. It has helped identify where laser damage of the capillaries may be an issue and possible ways this might be mitigated (e.g., addition of solenoid $B$-field).

\section{ACKNOWLEDGMENTS}

The authors wish to acknowledge Dr. Simon M. Hooker and Dr. Arie Zigler for their helpful comments and advice on capillary discharges. This work was supported by the U.S. Department of Energy, Grant No. DE-FG0204ER41294.

\section{APPENDIX A: BRAGINSKII TRANSPORT COEFFICIENTS IN THE 1D CAPILLARY MODEL}

Various transport coefficients $\alpha_{\|}^{\prime}, \alpha_{\perp}^{\prime}, \beta_{\Lambda}^{\prime \prime T}, \kappa_{i}^{\prime}, \kappa_{e}^{\prime}$, and $\eta_{\perp 0}$ are taken from Braginskii [17]. The following units will be employed: $R_{0}(\mathrm{~m}), n\left(\mathrm{~m}^{-3}\right), T(\mathrm{eV}), B_{\theta}(\mathrm{T})$, the plasma pressure $p\left(\mathrm{~J} / \mathrm{m}^{3}\right)$, the electron collision time with ions $\tau_{e, i}(\mathrm{~s})$, and $\eta(\mathrm{Ohm}-\mathrm{m})$. The electron collision time is

$$
\tau_{e}=3.44 \times 10^{11} T^{3 / 2} / n \ln \Lambda,
$$

where the Coulomb logarithm for hydrogen is

$$
\ln \Lambda=\left\{\begin{aligned}
29.9+\ln \left(T^{3 / 2} / n^{1 / 2} ;\right. & T<10 \mathrm{eV} \\
30.9+\ln \left(T / n^{1 / 2}\right) ; & T \geq 10 \mathrm{eV}
\end{aligned}\right\} .
$$

Since the model assumes charge and atomic mass equal to 1 and $T_{i}=T_{e}=T$, the ion collision time is longer by a fixed ratio $\tau_{i} / \tau_{e}=60.76$.

The electron magnetization is

$$
x_{e} \equiv\left(e B / m_{e}\right) \tau_{e}=1.760 \times 10^{11} B \tau_{e},
$$

where $B=\left(B_{\theta}^{2}+B_{z}^{2}\right)^{1 / 2}$. Note the ion magnetization is smaller by a fixed ratio $x_{i} / x_{e}=0.03332$ because $T_{i}=$ $T_{e}=T$.

Interspecies friction springs both from electron flow and electron temperature gradient. In Braginskii's terminology, the friction force density associated with electron flow is

$$
\mathbf{R}_{u}=-\alpha_{\|} \mathbf{u}_{e \|}-\alpha_{\perp} \mathbf{u}_{e \perp}+\alpha_{\Lambda} \mathbf{b} \times \mathbf{u}_{e} .
$$

We adopt dimensionless forms of the Braginskii's coefficients, i.e., $\alpha_{\|}^{\prime} \equiv \alpha_{\|} / e^{2} n^{2} \eta_{\perp 0}$ and $\beta_{\Lambda}^{\prime u T} \equiv\left(x_{e} / n\right) \beta_{\Lambda}^{u T}$. Then the velocity-related friction forces in the 1D capillary geometry are

$$
\begin{aligned}
& R_{u \theta} / e n=\eta_{\perp 0}\left[\left(\alpha_{\|}^{\prime} b_{\theta}^{2}+\alpha_{\perp}^{\prime} b_{z}^{2}\right) j_{\theta}+\left(\alpha_{\|}^{\prime}-\alpha_{\perp}^{\prime}\right) b_{\theta} b_{z} j_{z}\right], \\
& R_{u z} / e n=\eta_{\perp 0}\left[\left(\alpha_{\|}^{\prime}-\alpha_{\perp}^{\prime}\right) b_{\theta} b_{z} j_{\theta}+\left(\alpha_{\|}^{\prime} b_{z}^{2}+\alpha_{\perp}^{\prime} b_{\theta}^{2}\right) j_{z}\right] .
\end{aligned}
$$

Here the resistivity $\eta_{\perp 0}=m_{e} / e^{2} n \tau_{e}$ is the fully magnetized value.

In Braginskii's terminology, the friction force density associated with electron temperature gradient is

$$
\mathbf{R}_{T}=-\beta_{\|}^{u T} \nabla_{\|} k T_{e}-\beta_{\perp}^{u T} \nabla_{\perp} k T_{e}-\beta_{\Lambda}^{u T} \mathbf{b} \times \nabla k T_{e} .
$$

Then, the thermal friction forces are

$$
\begin{aligned}
\frac{R_{T \theta}}{e n} & =-\frac{\eta_{\perp 0}}{\mu_{0}} \frac{\beta}{4} \beta_{\Lambda}^{\prime u T} B_{z}\left(\frac{1}{T} \frac{d T}{d r}\right) ; \\
\frac{R_{T z}}{e n} & =\frac{\eta_{\perp 0}}{\mu_{0}} \frac{\beta}{4} \beta_{\Lambda}^{\prime u T} B_{\theta}\left(\frac{1}{T} \frac{d T}{d r}\right) .
\end{aligned}
$$

The coefficients are functions of the electron magnetization $x_{e} \equiv \omega_{c e} \tau_{e}$. For $Z=1$

$$
\begin{aligned}
\alpha_{\|}^{\prime} & =0.5129 \\
\alpha_{\perp}^{\prime} & =1-\left(6.416 x_{e}^{2}+1.837\right) / \Delta_{e} ; \\
\beta_{\Lambda}^{\prime \prime T} & =x_{e}^{2}\left(1.5 x_{e}^{2}+3.053\right) / \Delta_{e} \\
\Delta_{e} & =x_{e}^{4}+14.79 x_{e}^{2}+3.7703 \ldots
\end{aligned}
$$

Heat conduction can arise from the temperature gradient, and in the case of the electrons, from electron flow across the magnetic field. The heat flows from the temperature gradient for the two species are

$$
\begin{gathered}
\mathbf{q}_{T}^{e}=-\kappa_{\|}^{e} \nabla_{\|} T_{e}-\kappa_{\perp}^{e} \nabla_{\perp} T_{e}-\kappa_{\Lambda-}^{e} \mathbf{b} \times \nabla T_{e}, \\
\mathbf{q}_{T}^{i}=-\kappa_{\|}^{i} \nabla_{\|} T_{i}-\kappa_{\perp}^{i} \nabla_{\perp} T_{i}-\kappa_{\Lambda-}^{i} \mathbf{b} \times \nabla T_{i},
\end{gathered}
$$

where we have defined dimensionless forms of the thermal conduction coefficients using the fully magnetized resistivity, $\kappa_{\perp}^{e} / n k=\left(\eta_{\perp 0} / \mu_{0}\right) \kappa_{e}^{\prime}$ and $\kappa_{\perp}^{i} / n k=\left(\eta_{\perp 0} / \mu_{0}\right) \kappa_{i}^{\prime}$. Thus, the dimensionless conduction coefficients are

$$
\begin{gathered}
\kappa_{\perp}^{\prime e}=\frac{\beta}{4} \frac{x_{e}^{2}\left(4.664 x_{e}^{2}+11.92\right)}{\Delta_{e}}, \\
\kappa_{\perp}^{\prime i}=7.5543 \beta \frac{x_{i}^{2}\left(2 x_{i}^{2}+2.645\right)}{\Delta_{i}},
\end{gathered}
$$

where $\Delta_{i}=x_{i}^{4}+2.70 x_{i}^{2}+0.677$.

The thermal fluxes are then given by

$$
\begin{aligned}
& q_{T, r}^{e}=-\frac{\eta_{\perp 0}}{\mu_{0}} \kappa_{e}^{\prime} \frac{\beta}{4} \frac{B^{2}}{\mu_{0}}\left(\frac{1}{T} \frac{d T}{d r}\right) ; \\
& q_{T, r}^{i}=-\frac{\eta_{\perp 0}}{\mu_{0}} \kappa_{i}^{\prime} \frac{\beta}{4} \frac{B^{2}}{\mu_{0}}\left(\frac{1}{T} \frac{d T}{d r}\right) .
\end{aligned}
$$

Electron heat flux is also driven by electron flow perpendicular to the magnetic field. In Braginskii's terminology this is expressed as 


$$
\mathbf{q}_{u}^{e}=\beta_{\|}^{T u} \mathbf{u}_{\|}+\beta_{\perp}^{T u} \mathbf{u}_{\perp}+\beta_{\Lambda}^{T u} \mathbf{b} \times \mathbf{u} .
$$

This yields an electron flow-driven heat flux of

$$
\left(q_{u}^{e}\right)_{r}=\frac{\eta_{\perp 0}}{\mu_{0}} \frac{\beta}{4} \beta_{\Lambda}^{\prime u T}\left(-B_{\theta} j_{z}+B_{z} j_{\theta}\right) .
$$

\section{APPENDIX B: SIMPLE ANALYTIC MODEL OF CAPILLARY}

Besides the comprehensive, time-dependent model, the Bobrova paper [14] also included a quasianalytic approximation of the temperature profile in a capillary discharge. This quasianalytic model yields simple expressions linking the various design parameters. As such it offers a useful reference point for comparison with the numerical results from the more complete physical model presented here. We have slightly modified the Bobrova quasianalytic model by assuming the unmagnetized forms for both the electron thermal conductivity $\left(\kappa_{0} \propto T^{5 / 2}\right)$ and electrical resistivity $\left(\eta_{0} \propto T^{-3 / 2}\right)$, where $T$ is the local temperature. This is more consistent than the magnetized resistivity and unmagnetized conductivity assumed by Bobrova.

In the quasianalytic model, uniform pressure is assumed (no pinch effect) and the wall temperature is set to zero. This reduces the energy balance to an eigenvalue problem that can be solved by a single integration. The relationships between the various parameters are $T_{0}=0.17 \eta_{0} I^{2} / \kappa_{0} R_{0}^{2}$, $\langle n\rangle=1.55 n_{0}$, and $E_{z}=0.47 \eta_{0} I / R_{0}^{2}$. Then, the axis temperature and longitudinal electric field in MKS-eV units are given by the expressions

$$
\begin{gathered}
T_{0}=0.0123\left(I \ln \Lambda / R_{0}\right)^{2 / 5}, \\
E_{z}=0.0181(I \ln \Lambda)^{2 / 5} / R_{0}^{7 / 5} .
\end{gathered}
$$

As a representative example, if $I=300 \mathrm{~A}, \ln \Lambda=8, R_{0}=$ $250 \mu \mathrm{m}$, then $T_{0}=7.66 \mathrm{eV}$ and $E_{z}=45.0 \mathrm{kV} / \mathrm{m}$. The laser-channeling spot size is

$$
W=0.7424 \sqrt{\lambda_{p} R_{0}},
$$

where $\quad \lambda_{p}(\mu \mathrm{m})=3.34 \times 10^{10}\left[n_{e}\left(\mathrm{~cm}^{-3}\right)\right]^{-1 / 2}$ is the plasma wavelength. This expression is based on the assumption of an approximate parabolic density profile $n \approx$ $n_{0}\left[1+0.0334\left(r / R_{0}\right)^{2}+\cdots\right]$ near the axis for the analytic solution.

\section{APPENDIX C: LASER PROFILE FOR NONPARABOLIC DENSITY PROFILES}

The damage index depends on the electric field at the capillary wall. This can be found by solving Maxwell's equations. An electromagnetic wave propagating in a region with axisymmetric dielelectric constant $\epsilon(r)$ is gov- erned by

$$
\left(\nabla^{2}+\varepsilon \omega^{2} / c^{2}\right) \mathbf{E}+\nabla\left[\left(x E_{x}+y E_{y}\right) d(\ln \varepsilon) / d r\right]=0 .
$$

The second term, which involves gradients of $\epsilon(r)$, couples the field components so that a purely linearized polarization is not possible. However, the coupling is quite weak, as will be verified later. Ignoring this coupling, consider a nonevolving (constant spot size) laser beam with linear polarization $\mathbf{E}=E_{0} \hat{\mathbf{x}} \varphi(r) e^{i k z}$, where the profile function $\varphi$ is unity at the axis, $\varphi(0)=1$. Then

$$
\left[\frac{1}{r} \frac{d}{d r}\left(r \frac{d}{d r}\right)+\alpha^{2}\right] \varphi=0
$$

where the space-dependent radial structure parameter is $\alpha^{2}(r) \equiv \varepsilon(r) \omega^{2} / c^{2}-k^{2}$. The corresponding longitudinal field, $E_{z}=(i / k) E_{0}(x / r) d \varphi / d r$, has a maximum at $y=0$, $x=r$. The dielectric function for a plasma is $\epsilon=$ $1-n / n_{c}$, where $n_{c}=\pi / r_{e} \lambda^{2}$ is the critical density. For a purely parabolic density profile, the solution to Eq. (C2) is $\varphi=\exp \left(-r^{2} / W_{\mathrm{M}}^{2}\right)$.

The plasma region solution $\left(r \leq R_{0}\right)$ must be matched to the solution in the capillary material, which is governed by the same equation, but with a different and uniform dielectric constant $\varepsilon_{w}$. Suppose that the field is evanescent in the wall with a skin depth $\delta_{w} \ll R_{0}$. Then the wall solution is $\varphi=\varphi_{w} \exp \left[-\left(r-R_{0}\right) / \delta_{w}\right]$ and the longitudinal field for $x=r$ is $E_{z}=-\left(i / k \delta_{w}\right) E_{0} \varphi(r)$. The plasma and wall solutions are matched using jump conditions from Maxwell's equations $\left[\left[E_{z}\right]\right]=0$ and $\left[\left[\varepsilon E_{n}\right]\right]=0$, where $[[\cdots]]$ denotes the jump at $r=R_{0}$. This gives rise to an eigenvalue problem, which can be solved using a numerical algorithm based on the shooting method and a RungeKutta integrator. Representative results for the value of $D$ for non-Gaussian cases are presented in Sec. III C.

The coupled-polarization case, which includes the gradient term in Eq. (C1), was also solved approximately by treating the problem as if it were axisymmetric, replacing $x$ where it appears by $r$. The results for the damage index with polarization-coupling differed from the decoupled case by less than $0.1 \%$.

[1] P. Chen, J. M. Dawson, R. W. Huff, and T. Katsouleas, Phys. Rev. Lett. 54, 693 (1985).

[2] T. Tajima and J.M. Dawson, Phys. Rev. Lett. 43, 267 (1979); L. M. Gorbunov and V.I. Kirsanov, Sov. Phys. JETP 66, 290 (1987).

[3] V. Malka, S. Fritzler, E. Lefebvre, M.-M. Aleonard, F. Burgy, J.-P. Chambaret, J.-F. Chemin, K. Krushelnick, G. Malka, S. P. D. Mangles, Z. Najmudin, M. Pittman, J.-P. Rousseau, J.-N. Scheurer, B. Walton, and A. E. Dangor, Science 298, 1596 (2002).

[4] D. Kaganovich, P. V. Sasorov, Y. Ehrlich, C. Cohen, and A. Zigler, Appl. Phys. Lett. 71, 2925 (1997). 
[5] A. Butler, D. J. Spencer, and S. M. Hooker, Phys. Rev. Lett. 89, 185003 (2002).

[6] N.E. Andreev, L. M. Gorbunov, V.I. Kirsanov, A. A. Pogosova, and R. R. Ramazashvili, JETP Lett. 55, 571 (1992).

[7] P. Sprangle, E. Esarey, J. Krall, and G. Joyce, Phys. Rev. Lett. 69, 2200 (1992).

[8] T. M. Antonsen and P. Mora, Phys. Rev. Lett. 69, 2204 (1992).

[9] N.E. Andreev, S. V. Kuznetsov, A. A. Pogosova, L. C. Steinhauer, and W.D. Kimura, Phys. Rev. ST Accel. Beams 6, 041301 (2003).

[10] W. D. Kimura, N.E. Andreev, M. Babzien, I. Ben-Zvi, D. B. Cline, C. E. Dilley, S. C. Gottschalk, S. M. Hooker, K. P. Kusche, S. V. Kuznetsov, I. V. Pavlishin, I. V. Pogorelsky, A. A. Pogosova, L. C. Steinhauer, A. Ting, V. Yakimenko, A. Zigler, and F. Zhou, Phil. Trans. R. Soc. A 364, 611 (2006).

[11] Y. Ehrlich, C. Cohen, A. Zigler, J. Krall, P. Sprangle, and E. Esarey, Phys. Rev. Lett. 77, 4186 (1996).

[12] I. V. Pogorelsky, I. V. Pavlishin, I. Ben-Zvi, T. Kumita, Y. Kamiya, T. Hirose, B. Greenberg, D. Kaganovich,
A. Zigler, N. Andreev, N. Bobrova, and P. Sasorov, Appl. Phys. Lett. 83, 3459 (2003).

[13] W.D. Kimura, N.E. Andreev, M. Babzien, I. Ben-Zvi, D. B. Cline, C. E. Dilley, S. C. Gottschalk, S. M. Hooker, K.P. Kusche, S. V. Kuznetsov, R. H. Pantell, I. V. Pavlishin, I. V. Pogorelsky, A. A. Pogosova, L. C. Steinhauer, A. Ting, V. Yakimenko, A. Zigler, and F. Zhou, IEEE Trans. Plasma Sci. 33, 3 (2005).

[14] N. A. Bobrova, A. A. Esaulov, J.-I. Sakai, P. V. Sasorov, D. J. Spence, A. Butler, S. M. Hooker, and S. V. Bulanov, Phys. Rev. E 65, 016407 (2001).

[15] D. J. Spence and S. M. Hooker, J. Opt. Soc. Am. B 17, 1565 (2000).

[16] N. A. Bobrova, S. V. Bulanov, D. Farina, R. Pozzoli, T. L. Razinikova, J. Sakai, and P. V. Sasorov, J. Phys. Soc. Jpn. 67, 3437 (1998).

[17] S. I. Braginskii, Rev. Plasma Physics (I), edited by M. A. Leontovich (Consultants Bureau Enterprises, New York, 1965), p. 205.

[18] D. J. Spence and S. M. Hooker, Phys. Rev. E 63, 015401 (2000). 